\title{
The Characterization and Stability of g-Riesz Frames for Super Hilbert Space
}

\author{
Dingli Hua and Yongdong Huang \\ School of Mathematics and Information Science, Beifang University of Nationalities, Yinchuan 750021, China \\ Correspondence should be addressed to Yongdong Huang; nxhyd74@126.com
}

Received 12 July 2015; Accepted 10 September 2015

Academic Editor: Hans G. Feichtinger

Copyright (C) 2015 D. Hua and Y. Huang. This is an open access article distributed under the Creative Commons Attribution License, which permits unrestricted use, distribution, and reproduction in any medium, provided the original work is properly cited.

G-frames and g-Riesz frames as generalized frames in Hilbert spaces have been studied by many authors in recent years. The super Hilbert space has a certain advantage compared with the Hilbert space in the field of studying quantum mechanics. In this paper, for super Hilbert space $H \oplus K$, the definitions of a g-Riesz frame and minimal g-complete are put forward; also a characterization of $\mathrm{g}$-Riesz frames is obtained. In particular, we generalize them to general super Hilbert space $L_{1} \oplus L_{2} \oplus \cdots \oplus L_{n}$. Finally, a conclusion of the stability of a g-Riesz frame for the super Hilbert space is given.

\section{Introduction}

Frame as generalized basis in Hilbert space was first introduced by Duffin and Schaeffer [1] during the studied nonharmonic Fourier series in 1952. In 1986, Daubechies et al. [2] reintroduced the concept of frame. Now the theory of frames has been widely used in many areas such as the characterization of function spaces, signal processing, filter theory, image processing and quantum mechanics. We refer to [3-10] for an introduction to frame theory in Hilbert space and its application.

Sun [11] introduced g-Riesz basis and g-frame; g-frame actually generalized the concept of frame. Since then, gframe, g-frame sequence, Besselian g-frame, near g-Riesz bases, and so on are focused on and studied by many authors. The authors [12] introduced Besselian g-frames and near gRiesz bases in Hilbert space and gave some characterizations of them. In [13], g-Riesz frames were studied and some corresponding results were given. In [14], the concept of gbases in Hilbert spaces was introduced and some properties about g-bases were proved. Because super Hilbert spaces arose naturally as the state space of a quantum field in the functional Schrödinger representation of spinor quantum field theory and it provided a means to bring supersymmetric quantum field theories into a form resembling standard quantum mechanics, the super Hilbert space has certain advantages compared with the Hilbert space in quantum mechanics. With the extensive research of super Hilbert space [15-20], scholars were beginning to study g-frames for super Hilbert spaces [20,21]. Unfortunately, although g-Riesz frames were considered as a class of important frames, we have not consulted the literature of the g-Riesz frame for super Hilbert space $H \oplus K$ so far. Because g-Riesz frames play an important role in approximate calculating coefficients of g-frames, therefore, the study of g-Riesz frames for super Hilbert space $H \oplus K$ has a double meaning of theory and application. In order to enrich the frame theory, we give the concept of g-Riesz frame for super Hilbert space $H \oplus K$ and the characterization and necessary condition for g-Riesz frame. We also expand corresponding conclusions to general super Hilbert space $L_{1} \oplus L_{2} \oplus \cdots \oplus L_{n}$. Finally, we consider the stability of a g-Riesz frame for super Hilbert space.

Throughout this paper, $H$ and $K$ are two complex Hilbert spaces and $\left\{H_{j}: j \in J\right\}$ is a sequence of closed subspaces of $H$. $L\left(H, H_{j}\right)$ is the collection of all bounded linear operators 
from $H$ into $H_{j}$, where $J$ is a subset of integers $\mathbb{Z} . l^{2}\left(\left\{H_{j}\right\}_{j \in J}\right)$ is defined by

$$
\begin{aligned}
& l^{2}\left(\left\{H_{j}\right\}_{j \in J}\right) \\
& \quad=\left\{\left\{a_{j}\right\}_{j \in J}: a_{j} \in H_{j}, j \in J, \sum_{j \in J}\left\|a_{j}\right\|^{2}<+\infty\right\},
\end{aligned}
$$

with the inner product given by

$$
\left\langle\left\{a_{j}\right\}_{j \in J},\left\{b_{j}\right\}_{j \in J}\right\rangle=\sum_{j \in J}\left\langle a_{j}, b_{j}\right\rangle_{H_{j}},
$$

and it is clear that $l^{2}\left(\left\{H_{j}\right\}_{j \in J}\right)$ is a complex Hilbert space.

The literature [16] gave the definition of super Hilbert space.

Definition 1 (see [16, p.557]). Super Hilbert space is a direct sum that $H=H_{0} \oplus H_{1}$ of two complex Hilbert spaces $\left(H_{0},\langle\cdot, \cdot\rangle_{0}\right),\left(H_{1},\langle\cdot, \cdot\rangle_{1}\right)$ equipped with the super Hermitian form $\langle\langle\cdot, \cdot\rangle\rangle=\langle\cdot, \cdot\rangle_{0}+\langle\cdot, \cdot\rangle_{1}$.

\section{Preliminaries}

In this section, some necessary definitions and lemmas are introduced.

Definition 2 (see [11, Definition 1.1]). A sequence $\left\{\Lambda_{j} \in\right.$ $\left.L\left(H, H_{j}\right): j \in J\right\}$ is called a g-frame for $H$ with respect to $\left\{H_{j}\right\}_{j \in J}$ if there exist two positive constants $A$ and $B$ such that, for all $f \in H$,

$$
A\|f\|^{2} \leq \sum_{j \in J}\left\|\Lambda_{j} f\right\|^{2} \leq B\|f\|^{2}
$$

The constants $A$ and $B$ are called the lower and upper bounds of g-frame, respectively. If the right hand inequality holds, then we say that $\left\{\Lambda_{j}\right\}_{j \in J}$ is a g-Bessel sequence for $H$ with respect to $\left\{H_{j}\right\}_{j \in J}$. If $A=B$, we call this g-frame a tight gframe. If a g-frame ceases to be a g-frame whenever any single element is removed from $\left\{\Lambda_{j}\right\}_{j \in J}$, it is called an exact g-frame.

Definition 3 (see [14, Definition 2.2]). One says that $\left\{\Lambda_{j} \in\right.$ $\left.L\left(H, H_{j}\right): j \in J\right\}$ is g-complete, if $\left\{f \in H: \Lambda_{j} f=\right.$ 0 , for all $j\}=\{0\}$.

Definition 4 (see [11, Definition 3.1]). A sequence $\left\{\Lambda_{j} \in\right.$ $\left.L\left(H, H_{j}\right): j \in J\right\}$ is called a $g$-Riesz basis for $H$ with respect to $\left\{H_{j}\right\}_{j \in J}$, if the sequence $\left\{\Lambda_{j}\right\}_{j \in J}$ is g-complete and there exist positive constants $A$ and $B$ such that

$$
A \sum_{j \in J_{1}}\left\|g_{j}\right\|^{2} \leq\left\|\sum_{j \in J_{1}} \Lambda_{j}^{*} g_{j}\right\|^{2} \leq B \sum_{j \in J_{1}}\left\|g_{j}\right\|^{2}
$$

for all finite subset $J_{1} \subset J$ and $g_{j} \in H_{j}, j \in J_{1}$. The constants $A$ and $B$ are called the lower and upper bounds of g-Riesz bases, respectively.
Definition 5 (see [11, Definition 3.1]). Let $\left\{\Lambda_{j} \in L\left(H, H_{j}\right)\right.$ : $j \in J\}$. Suppose that $\left\{e_{j k}\right\}_{k \in K_{j}}$ is an orthonormal basis for $H_{j}$, where $K_{j}$ is a subset of $\mathbb{Z}$. Denote

$$
\begin{gathered}
u_{j k}=\Lambda_{j}^{*} e_{j k}, \quad j \in J, k \in K_{j}, \\
\Lambda_{j} f=\sum_{k \in K_{j}}\left\langle f, u_{j k}\right\rangle e_{j k}, \quad \forall f \in H .
\end{gathered}
$$

We call $\left\{u_{j k}\right\}_{j \in J, k \in K_{j}}$ the sequence induced by $\left\{\Lambda_{j}\right\}_{j \in J}$ with respect to $\left\{e_{j k}\right\}_{j \in J, k \in K_{j}}$.

Theorem 6 (see [21, Proposition 2.8]). Let $\left\{\Lambda_{j}\right\}_{j \in J}$ and $\left\{\Gamma_{j}\right\}_{j \in J}$ be sequences in $L\left(H, H_{j}\right)$ and $L\left(K, H_{j}\right)$, respectively, and let $\left\{e_{j k}\right\}_{k \in K_{j}}$ be an orthonormal basis for $H_{j}$, where $K_{j}$ is a subset of $\mathbb{Z}$, and let $\psi_{j k}=\Lambda_{j}^{*} e_{j k}, \varphi_{j k}=\Gamma_{j}^{*} e_{j k}$, and $\Theta_{j}(f, g)=$ $\Lambda_{j} f+\Gamma_{j} g$. Then $\left\{\left(\psi_{j k}, \varphi_{j k}\right)\right\}_{j \in J, k \in K_{j}}$ is a frame (resp., Bessel sequence, Riesz basis) for super Hilbert space $H \oplus K$ if and only if $\left.\left\{\Theta_{j} \in L\left(H \oplus K, H_{j}\right): j \in J\right)\right\}$ is a g-frame (resp., g-Bessel sequence, $g$-Riesz basis) for $H \oplus K$ with respect to $H_{j}$.

Proposition 7 (see [21, Proposition 2.9]). Let $\left\{\Theta_{j} \in L(H \oplus\right.$ $\left.\left.K, H_{j}\right): j \in J\right\},\left\{\left(\Lambda_{j} \in L\left(H, H_{j}\right): j \in J\right\}\right.$, and $\left\{\Gamma_{j} \in L\left(K, H_{j}\right):\right.$ $j \in J\}$ be $g$-frames, where $\Theta_{j}(f, g)=\Lambda_{j} f+\Gamma_{j} g$. Then $g$-frame operator for $\left\{\Theta_{j} \in L\left(H \oplus K, H_{j}\right): j \in J\right\}$ is defined by

$$
\begin{aligned}
& S_{\Theta}(f, g) \\
& \quad=\left(\sum_{j \in J}\left(\Lambda_{j}^{*} \Lambda_{j} f+\Lambda_{j}^{*} \Gamma_{j} g\right), \sum_{j \in J}\left(\Gamma_{j}^{*} \Lambda_{j} f+\Gamma_{j}^{*} \Gamma_{j} g\right)\right) .
\end{aligned}
$$

The literature [21] introduced the concept of disjoint gframes. A pair of g-frames $\left\{\Lambda_{j} \in L\left(H, H_{j}\right): j \in J\right\}$ and $\left\{\Gamma_{j} \in\right.$ $\left.L\left(K, H_{j}\right): j \in J\right\}$ is called disjoint if $\Theta_{j}(f, g)$ is a $g$-frame for $H \oplus K$, where $\Theta_{j}(f, g)=\Lambda_{j} f+\Gamma_{j} g$.

With the definition of g-complete of g-frame for Hilbert space, we give the definition of g-complete of g-frame for super Hilbert space as follows.

Definition 8. $\left\{\Theta_{j} \in L\left(H \oplus K, H_{j}\right)\right\}_{j=1}^{\infty}$ is called g-complete with respect to $\left\{H_{j}\right\}_{j=1}^{\infty}$ under the condition of that $\{(f, g)$ : $\Theta_{j}(f, g)=0$, for all $\left.j\right\}=\{(0,0)\}$.

\section{Characterization of g-Riesz Frame for Super Hilbert Space}

In this section, we first give the concept and the characterization of g-Riesz frame for super Hilbert space $H \oplus K$, and then we generalize them to super Hilbert space $L_{1} \oplus L_{2} \oplus \cdots \oplus L_{n}$.

3.1. Characterization of g-Riesz Frame for Super Hilbert Space $H \oplus K$. Before giving the characterization of g-Riesz frames for super Hilbert space $H \oplus K$, we give the definition of $g$-Riesz frames and some related lemmas. 
Suppose that $I$ is a subset of $J$, and denote

$$
(H \oplus K)_{I}=\overline{\left\{\sum_{j \in I_{1}} \Theta_{j}^{*} g_{j}=\left(\sum_{j \in I_{1}} \Lambda_{j}^{*} g_{j}, \sum_{j \in I_{1}} \Gamma_{j}^{*} g_{j}\right): \text { for any finite } I_{1} \subset I, g_{j} \in H_{j}, j \in I_{1}\right\} .}
$$

Definition 9. Suppose that $\left\{\Theta_{j} \in L\left(H \oplus K, H_{j}\right): j \in J\right\}$ is a g-frame for $H \oplus K$ with respect to $\left\{H_{j}\right\}_{j \in J}$. One says that $\left\{\Theta_{j}\right\}_{j \in J}$ is a g-Riesz frame if every subfamily $\left\{\Theta_{j}\right\}_{j \in I}$ of $\left\{\Theta_{j}\right\}_{j \in J}$ is a g-frame for $(H \oplus K)_{I}$ with respect to $\left\{H_{j}\right\}_{j \in I}$ with uniform g-frame lower bounds.

For the above $(H \oplus K)_{I}$, we have the following.

Lemma 10. Suppose that for every $j \in J, \Lambda_{j} \in L\left(H, H_{j}\right), \Gamma_{j} \in$ $L\left(K, H_{j}\right), \Theta_{j} \in L\left(H \oplus K, H_{j}\right)$, and $\left\{e_{j k}\right\}_{k \in K_{j}}$ is an orthonormal basis for $H_{j} \cdot \psi_{j k}=\Lambda_{j}^{*} e_{j k}$ and $\varphi_{j m}=\Gamma_{j}^{*} e_{j m}$ are defined as in (5). Then $(H \oplus K)_{I}=\overline{\operatorname{span}}\left\{\left(\psi_{j k}, \varphi_{j m}\right)\right\}_{j \in I, k, m \in K_{j}}$.

Proof. Denote

$$
\begin{aligned}
& (\widehat{H \oplus K})_{I}=\left\{\sum_{j \in I_{1}} \Theta_{j}^{*} g_{j}\right. \\
& =\left(\sum_{j \in I_{1}} \Lambda_{j}^{*} g_{j}, \sum_{j \in I_{1}} \Gamma_{j}^{*} g_{j}\right): \text { for any finite } I_{1} \subset I, g_{j} \\
& \left.\in H_{j}, \quad j \in I_{1}\right\} .
\end{aligned}
$$

Since $\psi_{j k}=\Lambda_{j}^{*} e_{j k}, \varphi_{j m}=\Gamma_{j}^{*} e_{j m}, j \in I, k, m \in K_{j}$, we have $\left(\psi_{j k}, \varphi_{j m}\right) \in(\widehat{H \oplus K})_{I}, j \in I, k, m \in K_{j}$. This implies that $\operatorname{span}\left\{\left(\psi_{j k}, \varphi_{j m}\right)\right\}_{j \in I, k, m \in K_{j}} \subset(\widehat{H \oplus K})_{I}$. Therefore, $\overline{\operatorname{span}}\left\{\left(\psi_{j k}\right.\right.$, $\left.\left.\varphi_{j m}\right)\right\}_{j \in I, k, m \in K_{j}} \subset(H \oplus K)_{I}$.

On the other hand, suppose that $(f, g) \in(H \oplus K)_{I}$, and then there exists a finite subset $I_{1} \subset I$ and $g_{j} \in H_{j}, j \in I_{1}$, such that $(f, g)=\sum_{j \in I_{1}} \Theta_{j}^{*} g_{j}=\left(\sum_{j \in I_{1}} \Lambda_{j}^{*} g_{j}, \sum_{j \in I_{1}} \Gamma_{j}^{*} g_{j}\right)$. For every $j \in I_{1}$, let $g_{j}=\sum_{k \in K_{j}} c_{j k} e_{j k}$, where $\left\{c_{j k}\right\}_{k \in K_{j}} \in l^{2}$. Then, we have

$$
\begin{aligned}
\Theta_{j}^{*} g_{j} & =\left(\Lambda_{j}^{*} \sum_{k \in K_{j}} c_{j k} e_{j k}, \Gamma_{j}^{*} \sum_{k \in K_{j}} c_{j k} e_{j k}\right) \\
& =\left(\sum_{k \in K_{j}} c_{j k} \psi_{j k}, \sum_{k \in K_{j}} c_{j k} \varphi_{j k}\right) \\
& \in \overline{\operatorname{span}}\left\{\left(\psi_{j k}, \varphi_{j m}\right)\right\}_{j \in I, k, m \in K_{j}}
\end{aligned}
$$

Therefore,

$$
\begin{aligned}
(f, g) & =\left(\sum_{j \in I_{1}} \Lambda_{j}^{*} g_{j}, \sum_{j \in I_{1}} \Gamma_{j}^{*} g_{j}\right) \\
& \in \overline{\operatorname{span}}\left\{\left(\psi_{j k}, \varphi_{j m}\right)\right\}_{j \in I, k, m \in K_{j}} .
\end{aligned}
$$

This implies that $(H \oplus K)_{I} \subset \overline{\operatorname{span}}\left\{\left(\psi_{j k}, \varphi_{j m}\right)\right\}_{j \in I, k, m \in K_{j}}$. The proof of Lemma 10 is completed.

Lemma 11. Let $W$ be a closed subspace of $H \oplus K$ and $\left\{\Theta_{j} \in\right.$ $\left.L\left(H \oplus K, H_{j}\right)\right\}_{j \in J}$. Suppose that $\left(\psi_{j k}, \varphi_{j m}\right) \in W$ for every $j \in I, k, m \in K_{j}$, where $I$ is a subset of $J$ and $\left\{\psi_{j k}\right\}_{j \in J, k \in K_{j}}$, $\left\{\varphi_{j m}\right\}_{j \in J, m \in K_{j}}$ are defined as in (5). If $\left\{\Theta_{j}\right\}_{j \in I}$ is g-complete in $W$, then $\left\{\left(\psi_{j k}, \varphi_{j m}\right)\right\}_{j \in I, k, m \in K_{j}}$ is complete in $W$.

Proof. For any $j \in I, k, m \in K_{j}$, we have $\left(\psi_{j k}, \varphi_{j m}\right) \in W$, where $W$ is a closed subspace of $H \oplus K$. Furthermore, we obtain

$$
\overline{\operatorname{span}}\left\{\left(\psi_{j k}, \varphi_{j m}\right)\right\}_{j \in I, k, m \in K_{j}} \subset W .
$$

It is enough to prove that if $(f, g) \in W$ and $(f, g) \perp \overline{\operatorname{span}}\left\{\left(\psi_{j k}\right.\right.$, $\left.\left.\varphi_{j m}\right)\right\}_{j \in I, k, m \in K_{j}}$, then $\left\langle(f, g),\left(\psi_{j k}, \varphi_{j m}\right)\right\rangle=0$ for $j \in I, k, m \in$ $K_{j}$. By equality (6), we obtain

$$
\begin{aligned}
\Theta_{j}(f, g) & =\Lambda_{j} f+\Gamma_{j} g \\
& =\sum_{k \in K_{j}}\left\langle f, \psi_{j k}\right\rangle e_{j k}+\sum_{k \in K_{j}}\left\langle g, \varphi_{j k}\right\rangle e_{j k}=0,
\end{aligned}
$$

$$
\forall j \in I .
$$

Since $\left\{\Theta_{j}\right\}_{j \in I}$ is g-complete in $W$, we have $(f, g)=(0,0)$. Hence, $\left\{\left(\psi_{j k}, \varphi_{j m}\right)\right\}_{j \in J, k, m \in K_{j}}$ is complete in $W$.

Inspired by the concept of minimal g-complete of gframe for Hilbert space, we give the definition of minimal gcomplete of g-frame for super Hilbert space.

Definition 12. Let $W$ be a closed subspace of $H \oplus K$ and $I$ be a subset of $J$. If $\left\{\Theta_{j}\right\}_{j \in I}$ is g-complete in $W$, but $\left\{\Theta_{j}\right\}_{j \in I \backslash\left\{j_{0}\right\}}$ is not g-complete in $W$ for any $j_{0} \in I$, then one says that $\left\{\Theta_{j}\right\}_{j \in I}$ is minimal g-complete in $W$.

Lemma 13. Suppose that $\left\{\Theta_{j}\right\} \in L\left(H \oplus K, H_{j}\right)$ for $j \in J$ and $I$ is any finite nonempty subset of $J$. Then there exists 
a finite nonempty subset $I_{0} \subset I$ such that $\left\{\Theta_{j}\right\}_{j \in I_{0}}$ is minimal g-complete in $(H \oplus K)_{I_{0}}$ and $(H \oplus K)_{I_{0}}=(H \oplus K)_{I}$, where $(H \oplus K)_{I}$ is defined as in (8).

Proof. We prove Lemma 13 in two cases.

Case 1. $\left\{\Theta_{j}\right\}_{j \in I}$ is g-complete in $(H \oplus K)_{I}$ for any $j_{0} \in I$, but $\left\{\Theta_{j}\right\}_{j \in I \backslash\left\{j_{0}\right\}}$ is not g-complete in $(H \oplus K)_{I}$. Let $I_{0}=I$. Then the conclusion is right.

Case 2. Suppose that there exists $j_{0} \in I$ such that $\left\{\Theta_{j}\right\}_{j \in I \backslash\left\{j_{0}\right\}}$ is g-complete in $(H \oplus K)_{I}$. Let $I_{1}=I \backslash\left\{j_{0}\right\}$. If there still exists $j_{1} \in I_{1}$ such that $\left\{\left(\psi_{j k}, \varphi_{j m}\right)\right\}_{j \in I_{1} \backslash\left\{j_{1}\right\}, k, m \in K_{j}}$ is complete in $\overline{\operatorname{span}}\left\{\left(\psi_{j k}, \varphi_{j m}\right)\right\}_{j \in I, k, m \in K_{j}}$, then we remove $j_{2}$ from $I_{1}$ in the same way. Repeat the operation above. Because $I$ is nonempty and finite, this process must stop after finite steps. Assume that we remove $\left\{j_{0}, j_{1}, \ldots, j_{n}\right\}$ from $I$, where $n \in N$ and $I_{0}=I \backslash\left\{j_{0}, j_{1}, \ldots, j_{n}\right\}$. Then, we obtain that $I_{0} \subset I$ satisfies the following two statements:

$K)_{I}$.

(1) $\left\{\Theta_{j}\right\}_{j \in I_{0}}$ is g-complete in $(H \oplus K)_{I}$.

(2) For any $i_{0} \in I_{0},\left\{\Theta_{j}\right\}_{j \in I_{0} \backslash\left\{i_{0}\right\}}$ is not g-complete in $(H \oplus$

By Lemmas 10 and 11, the statement (1) implies that

(3) $\left\{\left(\psi_{j k}, \varphi_{j m}\right)\right\}_{j \in I_{0}, k, m \in K_{j}}$ is complete in $\overline{\operatorname{span}}\left\{\left(\psi_{j k}\right.\right.$, $\left.\left.\varphi_{j m}\right)\right\}_{j \in I, k, m \in K_{j}} \cdot$

Obviously, $I_{0}$ is nonempty. The proof is by contradiction.

Suppose $I_{0}=\emptyset$, by (1), we obtain that $\left\{\Theta_{j}\right\}_{j \in I_{0}}$ is gcomplete in $(H \oplus K)_{I}$. It is obvious that this is impossible. So $I_{0}$ is nonempty. Now we prove $(H \oplus K)_{I_{0}}=(H \oplus K)_{I}$. By Definition 12 and Lemma 10, $\left\{\Theta_{j}\right\}_{j \in I}$ is minimal g-complete in $\overline{\operatorname{span}}\left\{\left(\psi_{j k}, \varphi_{j m}\right)\right\}_{j \in I, k, m \in K_{j}}$. By (3), we get

$$
\begin{aligned}
\overline{\operatorname{span}} & \left\{\left(\psi_{j k}, \varphi_{j m}\right)\right\}_{j \in I_{0}, k, m \in K_{j}} \\
= & \overline{\operatorname{span}}\left\{\left(\psi_{j k}, \varphi_{j m}\right)\right\}_{j \in I, k, m \in K_{j}} .
\end{aligned}
$$

By Lemma 10, we get $(H \oplus K)_{I_{0}}=(H \oplus K)_{I}$. The proof of Lemma 13 is completed.

Based on this, we can obtain a characterization of g-Riesz frame for super Hilbert space $H \oplus K$.

Theorem 14. Let $\left\{\Theta_{j} \in L\left(H \oplus K, H_{j}\right): j \in J\right\}$ be a $g$ frame for $H \oplus K$ with respect to $\left\{H_{j}\right\}_{j \in J}$. Then the following two statements are equivalent. $\left\{H_{j}\right\}_{j \in J}$.

(1) $\left\{\Theta_{j}\right\}_{j \in J}$ is a g-Riesz frame for $H \oplus K$ with respect to

(2) There exists $A>0$ such that $\left\{\Theta_{j}\right\}_{j \in I}$ is minimal $g$ complete in $(H \oplus K)_{I}$ for any nonempty subset I of J. And

$$
A\|(f, g)\|^{2} \leq \sum_{j \in I}\left\|\Theta_{j}(f, g)\right\|^{2}, \quad \forall(f, g) \in(H \oplus K)_{I},
$$

where $(H \oplus K)_{I}$ is defined as in (8).

Proof. (1) $\Rightarrow(2)$. Since $\left\{\Theta_{j}\right\}_{j \in J}$ is a g-Riesz frame for $H \oplus K$ with respect to $\left\{H_{j}\right\}_{j \in J}$, there exists $A>0$ such that $\left\{\Theta_{j}\right\}_{j \in I}$ is a g-frame for $(H \oplus K)_{I}$ with respect to $\left\{H_{j}\right\}_{j \in I}$ for any nonempty $I$ of $J$. Then, we obtain

$$
A\|(f, g)\|^{2} \leq \sum_{j \in I}\left\|\Theta_{j}(f, g)\right\|^{2}, \quad \forall(f, g) \in(H \oplus K)_{I} .
$$

$(2) \Rightarrow(1)$. Suppose that $\left\{\Theta_{j}\right\}_{j \in J}$ is a g-frame for $H \oplus K$ with respect to $\left\{H_{j}\right\}_{j \in J}$ with upper bound $B$. Then

$$
\sum_{j \in J}\left\|\Theta_{j}(f, g)\right\|^{2} \leq B\|(f, g)\|^{2}, \quad \forall(f, g) \in H \oplus K .
$$

There are two cases to prove that $\left\{\Theta_{j}\right\}_{j \in I}$ is a g-frame for $(H \oplus K)_{I}$ with respect to $\left\{H_{j}\right\}_{j \in I}$, where $I$ is any nonempty subset of $J$.

Case 1. When $I$ is any finite nonempty subset of $J$, by Lemma 13, there exists a finite nonempty subset $I_{0} \subset I$ such that $\left\{\Theta_{j}\right\}_{j \in I_{0}}$ is minimal g-complete in $(H \oplus K)_{I_{0}}$ and $(H \oplus$ $K)_{I_{0}}=(H \oplus K)_{I}$. By (15), we have

$$
A\|(f, g)\|^{2} \leq \sum_{j \in I_{0}}\left\|\Theta_{j}(f, g)\right\|^{2}
$$

$$
\forall(f, g) \in(H \oplus K)_{I_{0}}=(H \oplus K)_{I} .
$$

Again by (15), for $\forall(f, g) \in(H \oplus K)_{I}$, we have

$$
\begin{aligned}
A\|(f, g)\|^{2} & \leq \sum_{j \in I_{0}}\left\|\Theta_{j}(f, g)\right\|^{2} \leq \sum_{j \in I}\left\|\Theta_{j}(f, g)\right\|^{2} \\
& \leq \sum_{j \in J}\left\|\Theta_{j}(f, g)\right\|^{2} \leq B\|(f, g)\|^{2} .
\end{aligned}
$$

Case 2. Let $I$ be any infinite subset of $J$ and $(f, g) \in(H \oplus K)_{I}$. Then, for any $\varepsilon>0$, there exists a finite subset $I_{1} \subset I$ and $g_{j} \in H_{j}, j \in I_{1}$, such that $\left\|(f, g)-\sum_{j \in I_{1}} \Theta_{j}^{*} g_{j}\right\| \leq \varepsilon$. Denote

$$
(H \oplus K)_{I_{1}}
$$

$$
=\overline{\left\{\left(\sum_{j \in I_{1}} \Lambda_{j}^{*} g_{j}, \sum_{j \in I_{1}} \Gamma_{j}^{*} g_{j}\right): \forall g_{j} \in H_{j}, j \in I_{1}\right\} .}
$$

Clearly, $(H \oplus K)_{I}=(H \oplus K)_{I_{1}} \oplus(H \oplus K)_{I_{1}}^{\perp}$. Suppose that there exists $\left(f_{1}, g_{1}\right) \in(H \oplus K)_{I_{1}}$ and $\left(f_{2}, g_{2}\right) \in(H \oplus K)_{I_{1}}^{\perp}$ such that $(f, g)-\left(\sum_{j \in I_{1}} \Lambda_{j}^{*} g_{j}, \sum_{j \in I_{1}} \Gamma_{j}^{*} g_{j}\right)=\left(f_{1}, g_{1}\right)+\left(f_{2}, g_{2}\right)$. By $\left\|(f, g)-\sum_{j \in I_{1}} \Theta_{j}^{*} g_{j}\right\| \leq \varepsilon$, we get $\left\|\left(f_{1}, g_{1}\right)\right\|^{2}+\left\|\left(f_{2}, g_{2}\right)\right\|^{2} \leq$ $\varepsilon^{2}$. Let $\left(f_{0}, g_{0}\right)=\left(\sum_{j \in I_{1}} \Lambda_{j}^{*} g_{j}, \sum_{j \in I_{1}} \Gamma_{j}^{*} g_{j}\right)+\left(f_{1}, g_{1}\right)$, we get $(f, g)=\left(f_{0}, g_{0}\right)+\left(f_{2}, g_{2}\right)$, where $\left(f_{0}, g_{0}\right) \in(H \oplus K)_{I_{1}}$. By Case 1, we have

$$
\begin{aligned}
A\|(f, g)\|^{2} & =A\left\|\left(f_{0}, g_{0}\right)\right\|^{2}+A\left\|\left(f_{2}, g_{2}\right)\right\|^{2} \\
& \leq \sum_{j \in I_{1}}\left\|\Theta_{j}\left(f_{0}, g_{0}\right)\right\|^{2}+A \varepsilon^{2} .
\end{aligned}
$$


Now we prove $\sum_{j \in I_{1}}\left\|\Theta_{j}(f, g)\right\|^{2}=\sum_{j \in I_{1}}\left\|\Theta_{j}\left(f_{0}, g_{0}\right)\right\|^{2}$. We only need to prove that $\Theta_{j}(f, g)=\Theta_{j}\left(f_{0}, g_{0}\right)$ for every $j \in I_{1}$. From (6), we obtain

$$
\begin{aligned}
\Theta_{j}(f, g) & =\sum_{k \in K_{j}}\left\langle(f, g),\left(\psi_{j k}, \varphi_{j k}\right)\right\rangle e_{j k} \\
& =\sum_{k \in K_{j}}\left\langle\left(f_{0}, g_{0}\right)+\left(f_{2}, g_{2}\right),\left(\psi_{j k}, \varphi_{j k}\right)\right\rangle e_{j k},
\end{aligned}
$$

$$
\forall j \in I_{1} .
$$

By Lemma 10, we have $\left(\psi_{j k}, \varphi_{j k}\right) \in(H \oplus K)_{I_{1}}$ for any $j \in I_{1}$, $k \in K_{j}$. From (22), we get

$$
\Theta_{j}(f, g)=\sum_{k \in K_{j}}\left\langle\left(f_{0}, g_{0}\right),\left(\psi_{j k}, \varphi_{j k}\right)\right\rangle e_{j k}, \quad \forall j \in I_{1} .
$$

Again by (6), we have

$$
\begin{aligned}
\Theta_{j}(f, g) & =\sum_{k \in K_{j}}\left\langle\left(f_{0}, g_{0}\right),\left(\psi_{j k}, \varphi_{j k}\right)\right\rangle e_{j k} \\
& =\Theta_{j}\left(f_{0}, g_{0}\right), \quad \forall j \in I_{1} .
\end{aligned}
$$

Using (21), we get

$$
\begin{aligned}
A\|(f, g)\|^{2} & \leq \sum_{j \in I_{1}}\left\|\Theta_{j}\left(f_{0}, g_{0}\right)\right\|^{2}+A \varepsilon^{2} \\
& =\sum_{j \in I_{1}}\left\|\Theta_{j}(f, g)\right\|^{2}+A \varepsilon^{2} \\
& \leq \sum_{j \in I}\left\|\Theta_{j}(f, g)\right\|^{2}+A \varepsilon^{2} \\
& \leq \sum_{j \in J}\left\|\Theta_{j}(f, g)\right\|^{2}+A \varepsilon^{2} \\
& \leq B\|(f, g)\|^{2}+A \varepsilon^{2} .
\end{aligned}
$$

Letting $\varepsilon \rightarrow 0, A\|(f, g)\|^{2} \leq \sum_{j \in I}\left\|\Theta_{j}(f, g)\right\|^{2} \leq B\|(f, g)\|^{2}$ for any $(f, g) \in(H \oplus K)_{I}$. The proof of Theorem 14 is completed.

\subsection{Characterization of g-Riesz Frame for Super Hilbert Space $L_{1} \oplus L_{2} \oplus \cdots \oplus L_{n}$}

Definition 15. Let $\left\{\Theta_{j} \in L\left(L_{1} \oplus L_{2} \oplus \cdots \oplus L_{n}, H_{j}\right): j \in J\right\}$ be a g-frame for $L_{1} \oplus L_{2} \oplus \cdots \oplus L_{n}$ with respect to $\left\{H_{j}\right\}_{j \in J}$. If any subsequence $\left\{\Theta_{j}\right\}_{j \in I, I C J}$ is also a $\mathrm{g}$-frame for $\left(L_{1} \oplus L_{2} \oplus \cdots \oplus L_{n}\right)_{I}$ with respect to $\left\{H_{j}\right\}_{j \in I}$ with uniform g-frame lower bound, then one says that $\left\{\Theta_{j}\right\}_{j \in J}$ is a g-Riesz frame for $L_{1} \oplus L_{2} \oplus$ $\cdots \oplus L_{n}$ with respect to $\left\{H_{j}\right\}_{j \in J}$.

Like (8), we can define $\left(L_{1} \oplus L_{2} \oplus \cdots \oplus L_{n}\right)_{I}$ as follows:

$$
\left(L_{1} \oplus L_{2} \oplus \cdots \oplus L_{n}\right)_{I}=\overline{\left\{\sum_{j \in I_{1}} \Theta_{j}^{*} g_{j}=\left(\sum_{j \in I_{1}} \Lambda_{j}^{(1) *} g_{j}, \sum_{j \in I_{1}} \Lambda_{j}^{(2) *} g_{j}, \ldots, \sum_{j \in I_{1}} \Lambda_{j}^{(n) *} g_{j}\right): I_{1} \subset I, \forall g_{j} \in H_{j}, j \in I_{1}\right\}},
$$

where $I_{1}$ is any finite subset of $I$ and $\left\{\Lambda_{j}^{(p)} \in L\left(L_{p}, H_{j}\right): j \in\right.$ $J\}, p=1,2, \ldots, n$. Similarly, we give two lemmas.

Lemma 16. For every $\left\{\Theta_{j} \in L\left(L_{1} \oplus L_{2} \oplus \cdots \oplus L_{n}, H_{j}\right)\right.$ : $j \in J\}$, let $\left\{e_{j k}\right\}_{k \in K_{j}}$ be an orthonormal basis of $H_{j}$ and $\psi_{j k_{p}}^{(p)}=$ $\Lambda_{j}^{(p) *} e_{j k_{p}}$, where $\left\{\Lambda_{j}^{(p)} \in L\left(L_{p}, H_{j}\right): j \in J\right\}, p=1,2, \ldots, n$. Then

$$
\begin{aligned}
& \left(L_{1} \oplus L_{2} \oplus \cdots \oplus L_{n}\right)_{I} \\
& \quad=\overline{\operatorname{span}}\left\{\left(\psi_{j k_{1}}^{(1)}, \psi_{j k_{2}}^{(2)}, \ldots, \psi_{j k_{n}}^{(n)}\right)\right\}_{j \in I, k_{1}, k_{2}, \ldots, k_{n} \in K_{j}} .
\end{aligned}
$$

Lemma 17. Let $W$ be a closed subspace of $L_{1} \oplus L_{2} \oplus \cdots \oplus L_{n}$ and let $\left\{e_{j k}\right\}_{k \in K_{j}}$ be an orthonormal basis of $H_{j}$. Suppose that I is a subset of $J$ and $\left\{\Theta_{j} \in L\left(L_{1} \oplus L_{2} \oplus \cdots \oplus L_{n}, H_{j}\right), j \in J\right\}$. For every $j \in I, k_{1}, k_{2}, \ldots, k_{n} \in K_{j}$, one has $\left(\psi_{j k_{1}}^{(1)}, \psi_{j k_{2}}^{(2)}, \ldots, \psi_{j k_{n}}^{(n)}\right) \in W$, where $\psi_{j k_{p}}^{(p)}=\Lambda_{j}^{(p) *} e_{j k_{p}}$. If $\left\{\Theta_{j}\right\}_{j \in I}$ is g-complete in $W$, then $\left\{\left(\psi_{j k_{1}}^{(1)}, \psi_{j k_{2}}^{(2)}, \ldots, \psi_{j k_{n}}^{(n)}\right)\right\}_{j \in I, k_{1}, k_{2}, \ldots, k_{n} \in K_{j}}$ is complete in $W$.
In terms of the concept of minimal g-complete of gframe for Hilbert space, we give the definition of minimal gcomplete of g-frame for super Hilbert space $L_{1} \oplus L_{2} \oplus \cdots \oplus L_{n}$.

Definition 18. Let $W$ be a closed subspace of $L_{1} \oplus L_{2} \oplus \cdots \oplus L_{n}$ and $I \subset J$. If $\left\{\Theta_{j}\right\}_{j \in I}$ is g-complete in $W$, but $\left\{\Theta_{j}\right\}_{j \in I \backslash\left\{j_{0}\right\}}$ is not g-complete in $W$ for any $j_{0} \in I$, and then one says that $\left\{\Theta_{j}\right\}_{j \in I}$ is minimal g-complete in $W$.

Lemma 19. Suppose that $\left\{\Theta_{j} \in L\left(L_{1} \oplus L_{2} \oplus \cdots \oplus L_{n}, H_{j}\right): j \in\right.$ $J\}$ and $I$ is any finite nonempty subset of $J$. Then there exists a finite nonempty subset $I_{0} \subset I$ such that $\left\{\Theta_{j}\right\}_{j \in I_{0}}$ is minimal g-complete in $\left(L_{1} \oplus L_{2} \oplus \cdots \oplus L_{n}\right)_{I_{0}}$ and

$$
\left(L_{1} \oplus L_{2} \oplus \cdots \oplus L_{n}\right)_{I_{0}}=\left(L_{1} \oplus L_{2} \oplus \cdots \oplus L_{n}\right)_{I},
$$

where $\left(L_{1} \oplus L_{2} \oplus \cdots \oplus L_{n}\right)_{I}$ is defined as (26).

Proof. The proof is similar to proof of Lemma 10. 
By above lemma, we can get a characterization of g-Riesz frame for super Hilbert space $L_{1} \oplus L_{2} \oplus \cdots \oplus L_{n}$. Obviously, Theorem 14 is the special case of Theorem 20.

Theorem 20. Let $\left\{\Theta_{j} \in L\left(L_{1} \oplus L_{2} \oplus \cdots \oplus L_{n}, H_{j}\right): j \in J\right\}$ be a g-frame for $L_{1} \oplus L_{2} \oplus \cdots \oplus L_{n}$ with respect to $\left\{H_{j}\right\}_{j \in J}$. Then the following two statements are equivalent.

(1) $\left\{\Theta_{j}\right\}_{j \in J}$ is a g-Riesz frame for $L_{1} \oplus L_{2} \oplus \cdots \oplus L_{n}$ with respect to $\left\{H_{j}\right\}_{j \in J}$.

(2) For any nonempty subset I of $J$, there exists $A>0$, if $\left\{\Theta_{j}\right\}_{j \in J}$ is minimal g-complete in $\left(L_{1} \oplus L_{2} \oplus \cdots \oplus L_{n}\right)_{I}$. And

$$
\begin{aligned}
A\left\|\left(f_{1}, f_{2}, \ldots, f_{n}\right)\right\|^{2} \leq \sum_{j \in I}\left\|\Theta_{j}\left(f_{1}, f_{2}, \ldots, f_{n}\right)\right\|^{2}, \\
\forall\left(f_{1}, f_{2}, \ldots, f_{n}\right) \in\left(L_{1} \oplus L_{2} \oplus \cdots \oplus L_{n}\right)_{I} .
\end{aligned}
$$

Proof. The proof is analogous to proof of Theorem 14.

\section{Stability of g-Riesz Frames for Super Hilbert Space $H \oplus K$}

In this section, we use the characterization of g-Riesz frame for super Hilbert space $H \oplus K$ in Section 3 to study the stability of g-Riesz frame for super Hilbert space $H \oplus K$.

The stability of $g$-frames is important in practice which is wildly studied by many authors; for example, see [22-24]. The following is a fundamental result in the study of the stability of g-frames.

Proposition 21 (see [23, Theorem 3.1]). Suppose that $\left\{\Lambda_{j}\right\}_{j \in J}$ is a g-frame for $H$ with respect to $\left\{H_{j}\right\}_{j \in J}$ with bounds $A$ and $B$. There exists $\lambda, \beta, \mu \geq 0$ such that $\max \{\lambda+\mu / \sqrt{A}, \beta\}<1$. If $\left\{\Gamma_{j} \in L\left(H, H_{j}\right)\right\}_{j \in J}$ satisfies

$$
\begin{aligned}
& \left(\sum_{j \in J}\left\|\left(\Lambda_{j}-\Gamma_{j}\right) f\right\|^{2}\right)^{1 / 2} \\
& \leq \lambda\left(\sum_{j \in J}\left\|\Lambda_{j} f\right\|^{2}\right)^{1 / 2}+\beta\left(\sum_{j \in J}\left\|\Gamma_{j} f\right\|^{2}\right)^{1 / 2} \\
& +\mu\|f\|
\end{aligned}
$$

for $f \in H$, then $\left\{\Gamma_{j}\right\}_{j \in J}$ is a g-frame for $H$ with respect to $\left\{H_{j}\right\}_{j \in J}$ with bounds

$$
\begin{aligned}
& \left(\frac{(1-\lambda) \sqrt{A}-\mu}{1+\beta}\right)^{2}, \\
& \left(\frac{(1+\lambda) \sqrt{B}+\mu}{1-\beta}\right)^{2} .
\end{aligned}
$$

Example 22 illustrates a g-Riesz frame of super Hilbert space $H \oplus K$ has no result of stability like Proposition 21 .
Example 22. Suppose that $\left\{\left(e_{j}, 0\right)\right\} \cup\left\{\left(0, e_{j}\right)\right\}$ is an orthonormal basis of $H \oplus H$, where $j \in J$ and $J=\mathbb{N}$. Let $H_{j}=$ $\overline{\operatorname{span}}\left\{e_{j}, e_{j+1}\right\}$. Define the bounded linear operator as follows:

$$
\Theta_{j}(f, f)=2 \Lambda_{j} f=2\left\langle f, e_{j}\right\rangle e_{j}, \quad \forall(f, f) \in H \oplus H .
$$

First, we prove that $\left\{\Theta_{j}\right\}_{j \in J}$ is a g-frame for $H \oplus H$ with respect to $\left\{H_{j}\right\}_{j \in J}$. In fact, for any $(f, f) \in H \oplus H$, we have

$$
\begin{aligned}
\sum_{j \in J}\left\|\Theta_{j}(f, f)\right\|^{2} & =\sum_{j \in J}\left\|2\left\langle f, e_{j}\right\rangle e_{j}\right\|^{2} \\
& =4 \sum_{j \in J}\left\|\left\langle f, e_{j}\right\rangle e_{j}\right\|^{2}=4\|f\|^{2} \\
& =2\|(f, f)\|^{2} .
\end{aligned}
$$

Thus, $\left\{\Theta_{j}\right\}_{j \in J}$ is a g-frame for $H \oplus H$ with respect to $\left\{H_{j}\right\}_{j \in J}$. For any $g_{j} \in H_{j}$, let $g_{j}=c_{j} e_{j}+c_{j+1} e_{j+1}$, and we have

$$
\begin{aligned}
\left\langle\Theta_{j}^{*} g_{j},(f, f)\right\rangle & =\left\langle g_{j}, \Theta_{j}(f, f)\right\rangle=\left\langle g_{j}, 2 \Lambda_{j} f\right\rangle \\
& =\left\langle c_{j} e_{j}+c_{j+1} e_{j+1}, 2\left\langle f, e_{j}\right\rangle e_{j}\right\rangle \\
& =2 c_{j} \overline{\left\langle f, e_{j}\right\rangle}=2 c_{j}\left\langle e_{j}, f\right\rangle \\
& =\left\langle\left(c_{j} e_{j}, c_{j} e_{j}\right),(f, f)\right\rangle .
\end{aligned}
$$

It implies that $\Theta_{j}^{*} g_{j}=\left(c_{j} e_{j}, c_{j} e_{j}\right)$. Since $\left\{e_{j}, e_{j+1}\right\}$ is an orthonormal basis for $H_{j}$, we have

$$
\begin{aligned}
& \psi_{j_{1}}=\Lambda_{j}^{*} e_{j}=e_{j}, \\
& \psi_{j_{2}}=\Lambda_{j}^{*} e_{j+1}=0 .
\end{aligned}
$$

So $\left\{\left(\psi_{j k}, \psi_{j m}\right)\right\}_{j \in J, k, m \in K_{j}}=\left\{\left(e_{j}, 0\right),\left(0, e_{j}\right),(0,0),\left(e_{j}, e_{j}\right)\right\}_{j=1}^{\infty}$.

Next, we prove that $\left\{\Theta_{j}\right\}_{j \in J}$ is a g-Riesz frame for $H \oplus H$ with respect to $\left\{H_{j}\right\}_{j \in J}$. Let $I$ be any finite subset of $J$. From Lemma 10, we obtain that

$$
\begin{aligned}
(H \oplus H)_{I} & =\overline{\operatorname{span}}\left\{\left(\psi_{j k}, \psi_{j m}\right)\right\}_{j \in I, k, m \in K_{j}} \\
& =\overline{\operatorname{span}}\left\{\left(e_{j}, 0\right),\left(0, e_{j}\right),(0,0),\left(e_{j}, e_{j}\right)\right\}_{j \in I} .
\end{aligned}
$$

Hence, for every $j_{0} \in J$, we have $\overline{\operatorname{span}}\left\{\left(e_{j}, 0\right),\left(0, e_{j}\right)\right\}_{j \in I \backslash\left\{j_{0}\right\}} \neq$ $\overline{\operatorname{span}}\left\{\left(e_{j}, 0\right),\left(0, e_{j}\right)\right\}_{j \in I}$. Therefore, $\left\{\left(\psi_{j k}, \psi_{j m}\right)\right\}_{j \in I, k, m \in K_{j}}$ is minimal g-complete in $(H \oplus H)_{I}$. Now we prove that $\left\{\Theta_{j}\right\}_{j \in I}$ is minimal g-complete in $(H \oplus H)_{I}$.

If there exists $(f, f) \in(H \oplus H)_{I}$ such that $\Theta_{j}(f, f)=$ $2 \Lambda_{j} f=0$, then we have

$$
\begin{aligned}
0 & =\Theta_{j}(f, f)=2 \Lambda_{j} f=2\left\langle f, e_{j}\right\rangle e_{j} \\
& =2\left\langle(f, f),\left(e_{j}, 0\right)\right\rangle e_{j}, \\
0 & =\Theta_{j}(f, f)=2 \Lambda_{j} f=2 \overline{\left\langle e_{j}, f\right\rangle} e_{j} \\
& =2 \overline{\left(\langle 0, f\rangle+\left\langle e_{j}, f\right\rangle\right)} e_{j}=2 \overline{\left\langle\left(0, e_{j}\right),(f, f)\right\rangle} e_{j} .
\end{aligned}
$$


Since $\left\{e_{j}, e_{j+1}\right\}$ is an orthonormal basis for $H_{j}$, we have $\langle(f, f)$, $\left.\left(e_{j}, 0\right)\right\rangle=0$ and $\overline{\left\langle\left(0, e_{j}\right),(f, f)\right\rangle}=0$. From the minimal complete of $\left\{\left(\psi_{j k}, \psi_{j m}\right)\right\}_{j \in I}$ for $k, m \in K_{j}$ in $(H \oplus H)_{I}$ and $\overline{\operatorname{span}}\left\{\left(\psi_{j k}, \psi_{j m}\right)\right\}_{j \in I, k, m \in K_{j}}=\overline{\operatorname{span}}\left\{\left(e_{j}, 0\right),\left(0, e_{j}\right)\right\}_{j \in I}$, we can obtain $(f, f)=(0,0)$. This implies that $\left\{\Theta_{j}\right\}_{j \in I}$ is minimal g-complete in $(H \oplus H)_{I}$. Then, for any $(f, f) \in(H \oplus H)_{I}$, we have

$$
\sum_{j \in I}\left\|\Theta_{j}(f, f)\right\|^{2}=\sum_{j \in I}\left\|2\left\langle f, e_{j}\right\rangle e_{j}\right\|^{2}=2\|(f, f)\|^{2} .
$$

By Theorem 14, $\left\{\Theta_{j}\right\}_{j \in J}$ is a g-Riesz frame for $H \oplus H$ with respect to $\left\{H_{j}\right\}_{j \in J}$.

Let $\varepsilon \in(0,1)$. Define the bounded linear operator $\Omega_{j}$ : $H \oplus H \rightarrow H_{j}$ as follows:

$$
\Omega_{j}(f, f)=\Theta_{j}(f, f)+\frac{2 \varepsilon}{j+1}\left\langle f, e_{j+1}\right\rangle e_{j+1} .
$$

By direct calculation, for any $g_{j} \in H_{j}$, suppose that $g_{j}=c_{j} e_{j}+$ $c_{j+1} e_{j+1}$, and we have $\Omega_{j}^{*} g_{j}=\left(c_{j} e_{j}+(\varepsilon /(j+1)) c_{j+1} e_{j+1}, c_{j} e_{j}+\right.$ $\left.(\varepsilon /(j+1)) c_{j+1} e_{j+1}\right)$. Let $\widetilde{\psi}_{j_{1}}=e_{j}, \widetilde{\psi}_{j_{2}}=(\varepsilon /(j+1)) e_{j+1}$. Then

$$
\begin{gathered}
\left\{\left(\tilde{\psi}_{j k}, \tilde{\psi}_{j m}\right)\right\}_{j \in J, k, m \in K_{j}}=\left\{\left(e_{j}, \frac{\varepsilon}{j+1} e_{j+1}\right),\left(e_{j}, e_{j}\right),\right. \\
\left.\left(\frac{\varepsilon}{j+1} e_{j+1}, \frac{\varepsilon}{j+1} e_{j+1}\right),\left(\frac{\varepsilon}{j+1} e_{j+1}, e_{j}\right)\right\}_{j=1}^{\infty} .
\end{gathered}
$$

Now we prove that $\left\{\Omega_{j}\right\}_{j \in J}$ is not a g-Riesz frame for $H \oplus H$ with respect to $\left\{H_{j}\right\}_{j \in J}$.

The proof is by contradiction. Suppose that $\left\{\Omega_{j}\right\}_{j \in J}$ is a gRiesz frame for $H \oplus H$ with respect to $\left\{H_{j}\right\}_{j \in J}$. Let $I=\{j\}$. By Lemma 10, we have

$$
\begin{aligned}
(H & \oplus H)_{I}=\overline{\operatorname{span}}\left\{\left(\psi_{j k}, \psi_{j m}\right)\right\}_{j \in I, k, m \in K_{j}} \\
& =\overline{\operatorname{span}}\left\{\left(e_{j}, \frac{\varepsilon}{j+1} e_{j+1}\right),\left(e_{j}, e_{j}\right),\right. \\
& \left.\left(\frac{\varepsilon}{j+1} e_{j+1}, \frac{\varepsilon}{j+1} e_{j+1}\right),\left(\frac{\varepsilon}{j+1} e_{j+1}, e_{j}\right)\right\} \\
& =\overline{\operatorname{span}}\left\{\left(e_{j}, e_{j}\right),\left(e_{j}, e_{j+1}\right),\left(e_{j+1}, e_{j}\right),\left(e_{j+1}, e_{j+1}\right)\right\} .
\end{aligned}
$$

Choosing $\left(e_{j+1}, e_{j+1}\right) \in(H \oplus H)_{I}$, then

$$
\left\|\Omega_{j}\left(e_{j+1}, e_{j+1}\right)\right\|^{2}=\frac{4 \varepsilon^{2}}{(j+1)^{2}} .
$$

Since $\left\{\Omega_{j}\right\}_{j \in I}$ is a g-frame for $(H \oplus H)_{I}$ with respect to $\left\{H_{j}\right\}_{j \in I}$, there exists $A>0$ such that

$$
\begin{aligned}
A & =A\left\|\left(e_{j+1}, e_{j+1}\right)\right\|^{2} \leq \sum_{k \in I}\left\|\Omega_{k}\left(e_{j+1}, e_{j+1}\right)\right\|^{2} \\
& =\left\|\Omega_{j}\left(e_{j+1}, e_{j+1}\right)\right\|^{2}=\frac{4 \varepsilon^{2}}{(j+1)^{2}} .
\end{aligned}
$$

Letting $j \rightarrow+\infty$, it implies that $A=0$. But this is a contradiction. We conclude that $\left\{\Omega_{j}\right\}_{j \in J}$ is not a g-Riesz frame for $H \oplus H$ with respect to $\left\{H_{j}\right\}_{j \in J}$.

On the other hand, for any $(f, f) \in H \oplus H$, we have

$$
\begin{aligned}
& \left(\sum_{j \in J}\left\|\left(\Theta_{j}-\Omega_{j}\right)(f, f)\right\|^{2}\right)^{1 / 2} \\
& =\left(\sum_{j \in J}\left\|\frac{2 \varepsilon}{j+1}\left\langle f, e_{j+1}\right\rangle e_{j+1}\right\|^{2}\right)^{1 / 2} \leq 2 \varepsilon\|(f, f)\| .
\end{aligned}
$$

From Example 22, we can realize that $\lambda, \beta=0, \mu \neq 0$. Suppose that $\left\{\Theta_{j}\right\}_{j \in J}$ is a g-Riesz frame for $H \oplus K$ with respect to $\left\{H_{j}\right\}_{j \in J}$. Even if $\left\{\Theta_{j}\right\}_{j \in J}$ and $\left\{\Omega_{j}\right\}_{j \in J}$ satisfy the inequality of Proposition 21, it is uncertain to get that $\left\{\Omega_{j}\right\}_{j \in J}$ is a g-Riesz frame for $H \oplus K$ with respect to $\left\{H_{j}\right\}_{j \in J}$.

Theorem 23. Let $\left\{\Theta_{j}\right\}_{j \in J}$ be a g-Riesz frame for $H \oplus K$ with respect to $\left\{H_{j}\right\}_{j \in J}$ with bounds $B$ and $A, \lambda, \beta \in[0,1)$. If $\left\{\Omega_{j} \in\right.$ $\left.L\left(H \oplus K, H_{j}\right)\right\}_{j \in J}$ satisfies

$$
\begin{gathered}
\left(\sum_{j \in J_{1}}\left\|\left(\Theta_{j}-\Omega_{j}\right)(f, g)\right\|^{2}\right)^{1 / 2} \\
\leq \lambda\left(\sum_{j \in J_{1}}\left\|\Theta_{j}(f, g)\right\|^{2}\right)^{1 / 2} \\
+\beta\left(\sum_{j \in J_{1}}\left\|\Omega_{j}(f, g)\right\|^{2}\right)^{1 / 2}
\end{gathered}
$$

for any $(f, g) \in H \oplus K$, where $J_{1}$ is any finite subset of $J$, then $\left\{\Omega_{j}\right\}_{j \in J}$ is a g-Riesz frame for $H \oplus K$ with respect to $\left\{H_{j}\right\}_{j \in J}$ with bounds

$$
\begin{aligned}
& A\left(\frac{1-\lambda}{1+\beta}\right)^{2}, \\
& B\left(\frac{1+\lambda}{1-\beta}\right)^{2} .
\end{aligned}
$$

Proof. Since $\left\{\Theta_{j}\right\}_{j \in J}$ is a g-frame for $H \oplus K$ with respect to $\left\{H_{j}\right\}_{j \in J}$ with bounds $A$ and $B$, for any finite subset $J_{1} \subset J$, then

$$
\begin{aligned}
\sum_{j \in J_{1}}\left\|\Theta_{j}(f, g)\right\|^{2} \leq \sum_{j \in J}\left\|\Theta_{j}(f, g)\right\|^{2} \leq B\|(f, g)\|^{2}, \\
\forall(f, g) \in H \oplus K .
\end{aligned}
$$

By the triangle inequality, we have

$$
\begin{gathered}
\left(\sum_{j \in J_{1}}\left\|\left(\Theta_{j}-\Omega_{j}\right)(f, g)\right\|^{2}\right)^{1 / 2} \\
\geq\left(\sum_{j \in J_{1}}\left\|\Omega_{j}(f, g)\right\|^{2}\right)^{1 / 2} \\
-\left(\sum_{j \in J_{1}}\left\|\Theta_{j}(f, g)\right\|^{2}\right)^{1 / 2} .
\end{gathered}
$$


From (45), (47), and (48), we have

$$
\begin{aligned}
& \left(\sum_{j \in J_{1}}\left\|\Omega_{j}(f, g)\right\|^{2}\right)^{1 / 2} \\
& \leq\left(\sum_{j \in J_{1}}\left\|\left(\Theta_{j}-\Omega_{j}\right)(f, g)\right\|^{2}\right)^{1 / 2} \\
& +\left(\sum_{j \in J_{1}}\left\|\Theta_{j}(f, g)\right\|^{2}\right)^{1 / 2} \\
& \leq \frac{\lambda+1}{1-\beta}\left(\sum_{j \in J_{1}}\left\|\Theta_{j}(f, g)\right\|^{2}\right)^{1 / 2} \\
& \leq \frac{(\lambda+1) \sqrt{B}}{1-\beta}\|(f, g)\| .
\end{aligned}
$$

Therefore, the series $\sum_{j \in J_{1}}\left\|\Omega_{j}(f, g)\right\|^{2}$ is convergent. By (45), we have

$$
\begin{gathered}
\left(\sum_{j \in J}\left\|\left(\Theta_{j}-\Omega_{j}\right)(f, g)\right\|^{2}\right)^{1 / 2} \\
\leq \lambda\left(\sum_{j \in J}\left\|\Theta_{j}(f, g)\right\|^{2}\right)^{1 / 2} \\
+\beta\left(\sum_{j \in J}\left\|\Omega_{j}(f, g)\right\|^{2}\right)^{1 / 2} .
\end{gathered}
$$

By Proposition 21, we get that $\left\{\Omega_{j}\right\}_{j \in J}$ is a g-frame for $H \oplus K$ with respect to $\left\{H_{j}\right\}_{j \in J}$. For any finite subset $J_{1} \subset J$, by the triangle inequality, we obtain

$$
\begin{gathered}
\left(\sum_{j \in J_{1}}\left\|\left(\Theta_{j}-\Omega_{j}\right)(f, g)\right\|^{2}\right)^{1 / 2} \\
\geq\left(\sum_{j \in J_{1}}\left\|\Theta_{j}(f, g)\right\|^{2}\right)^{1 / 2} \\
-\left(\sum_{j \in J_{1}}\left\|\Omega_{j}(f, g)\right\|^{2}\right)^{1 / 2} .
\end{gathered}
$$

Using (45) and (51), we have

$$
\begin{aligned}
& \left(\sum_{j \in J_{1}}\left\|\Theta_{j}(f, g)\right\|^{2}\right)^{1 / 2} \\
& \leq \frac{1+\beta}{1-\lambda}\left(\sum_{j \in J_{1}}\left\|\Omega_{j}(f, g)\right\|^{2}\right)^{1 / 2} .
\end{aligned}
$$

For any finite subset $I \subset J$, denote

$$
U_{I}=\overline{\left\{\sum_{j \in I} \Omega_{j}^{*} g_{j}: \forall g_{j} \in H_{j}, j \in I\right\} .}
$$

If $\left\{\Omega_{j}\right\}_{j \in I}$ is minimal g-complete in $U_{I}$, we need to prove that $U_{I} \subset(H \oplus K)_{I}$; that is, $(H \oplus K)_{I}^{\perp} \subset U_{I}^{\perp}$. For any $(f, g) \in(H \oplus K)_{I}^{\perp}$, since $H \oplus K=U_{I} \oplus U_{I}^{\perp}$, there exist $\left(f_{1}, g_{1}\right) \in U_{I}$ and $\left(f_{2}, g_{2}\right) \in U_{I}^{\perp}$ such that $(f, g)=$ $\left(f_{1}, g_{1}\right)+\left(f_{2}, g_{2}\right)$. From Lemma 10 , we obtain that $(H \oplus K)_{I}=$ $\overline{\operatorname{span}}\left\{\left(\psi_{j k}, \varphi_{j m}\right)\right\}_{j \in I, k, m \in K_{j}}$ and $U_{I}=\overline{\operatorname{span}}\left\{\left(\widetilde{\psi}_{j k}, \widetilde{\varphi}_{j m}\right)\right\}_{j \in I, k, m \in K_{j}}$, where $\widetilde{\psi}_{j k}=\Lambda_{j}^{*} e_{j k}, \widetilde{\varphi}_{j m}=\Gamma_{j}^{*} e_{j m}$ for $j \in I, k, m \in K_{j}$. Therefore, for any finite $j \in I$, we have

$$
\begin{aligned}
\Theta_{j}(f, g) & =\sum_{k \in K_{j}}\left\langle(f, g),\left(\psi_{j k}, \varphi_{j k}\right)\right\rangle e_{j k}=0, \\
\Omega_{j}(f, g) & =\sum_{k \in K_{j}}\left\langle(f, g),\left(\tilde{\psi}_{j k}, \widetilde{\varphi}_{j k}\right)\right\rangle e_{j k} \\
& =\sum_{k \in K_{j}}\left\langle\left(f_{1}, g_{1}\right)+\left(f_{2}, g_{2}\right),\left(\widetilde{\psi}_{j k}, \widetilde{\varphi}_{j k}\right)\right\rangle e_{j k} \\
& =\sum_{k \in K_{j}}\left\langle\left(f_{1}, g_{1}\right),\left(\tilde{\psi}_{j k}, \widetilde{\varphi}_{j k}\right)\right\rangle e_{j k} \\
& =\Omega_{j}\left(f_{1}, g_{1}\right) .
\end{aligned}
$$

By (45), we have $\left(\sum_{j \in I}\left\|\Omega_{j}(f, g)\right\|^{2}\right)^{1 / 2} \leq \beta\left(\sum_{j \in I} \| \Omega_{j}\left(f_{1}\right.\right.$, $\left.\left.g_{1}\right) \|^{2}\right)^{1 / 2}$.

Then for any $j \in I$, it follows that $\Omega_{j}\left(f_{1}, g_{1}\right)=0$. Since $\left\{\Omega_{j}\right\}_{j \in I}$ is g-complete in $U_{I}$, we have $\left(f_{1}, g_{1}\right)=0$. Therefore $(f, g)=\left(f_{2}, g_{2}\right) \in U_{I}^{\perp}$, and it implies that $(H \oplus K)_{I}^{\perp} \subset U_{I}^{\perp}$.

For any $f \in U_{I} \subset(H \oplus K)_{I}$, since $\left\{\Theta_{j}\right\}_{j \in I}$ is a g-frame for $(H \oplus K)_{I}$ with respect to $\left\{H_{j}\right\}_{j \in I}$ with lower bound $A$, by (52), we have

$$
\begin{aligned}
A\left(\frac{1-\lambda}{1+\beta}\right)^{2}\|(f, g)\|^{2} \leq \sum_{j \in I}\left\|\Omega_{j}(f, g)\right\|^{2} & \\
& \\
& \forall(f, g) \in U_{I} .
\end{aligned}
$$

By Theorem 14, we get that $\left\{\Omega_{j}\right\}_{j \in J}$ is a g-Riesz frame for $H \oplus K$ with respect to $\left\{H_{j}\right\}_{j \in J}$. The proof of Theorem 23 is completed.

\section{Conflict of Interests}

The authors declare that there is no conflict of interests regarding the publication of this paper.

\section{Acknowledgments}

This work is supported by National Natural Science Foundation of China (Grants nos. 61261043 and 10961001), Natural Science Foundation of Ningxia (Grant no. NZ13084), and Innovation Project for Graduate of Beifang University of Nationalities. 


\section{References}

[1] R. J. Duffin and A. C. Schaeffer, "A class of nonharmonic Fourier series," Transactions of the American Mathematical Society, vol. 72, pp. 341-366, 1952.

[2] I. Daubechies, A. Grossmann, and Y. Meyer, "Painless nonorthogonal expansions," Journal of Mathematical Physics, vol. 27, no. 5, pp. 1271-1283, 1986.

[3] O. Agratini and O. Dŏgru, "Weighted approximation by gSzász-King type operators," Taiwanese Journal of Mathematics, vol. 14, no. 4, pp. 1283-1296, 2010.

[4] H. Bölcskei, F. Hlawatsch, and H. G. Feichtinger, "Frametheoretic analysis of oversampled filter banks," IEEE Transactions on Signal Processing, vol. 46, no. 12, pp. 3256-3268, 1998.

[5] E. J. Candès and D. L. Donoho, "New tight frames of curvelets and optimal representations of objects with piecewise $C^{2}$ singularities," Communications on Pure and Applied Mathematics, vol. 57, no. 2, pp. 216-266, 2004.

[6] P. G. Casazza, "Characterizing Hilbert space frames with the subframe property," Illinois Journal of Mathematics, vol. 41, no. 4, pp. 648-666, 1997.

[7] X. Guo, "Operator characterizations and some properties of g-frames on hilbert spaces," Journal of Function Spaces and Applications, vol. 2013, Article ID 931367, 9 pages, 2013.

[8] O. Christensen, An Introduction to Frames and Riesz Bases, Birkhäuser, Boston, Mass, USA, 2003.

[9] J. R. Holub, "Pre-frame operators, Besselian frames, and nearRiesz bases in Hilbert spaces," Proceedings of the American Mathematical Society, vol. 122, no. 3, pp. 779-785, 1994.

[10] R. M. Young, An Introduction to Nonharmonic Fourier Series, Academic Press, New York, NY, USA, 2001.

[11] W. Sun, "G-frames and g-Riesz bases," Journal of Mathematical Analysis and Applications, vol. 322, no. 1, pp. 437-452, 2006.

[12] M. R. Abdollahpour and A. Najati, "Besselian g-frames and near g-Riesz bases," Applicable Analysis and Discrete Mathematics, vol. 5, no. 2, pp. 259-270, 2011.

[13] Z. Li and Y. Zhu, "G-Riesz frames in Hilbert spaces," Science China Mathematics, Chinese Series, vol. 41, no. 1, pp. 53-68, 2011.

[14] X. Guo, "g-bases in Hilbert spaces," Abstract and Applied Analysis, vol. 2012, Article ID 923729, 14 pages, 2012.

[15] B. DeWitt, Supermanifolds, Cambridge Monographs on Mathematical Physics, Cambridge University Press, New York, NY, USA, 2nd edition, 1992.

[16] A. M. El Gradechi and L. M. Nieto, "Supercoherent states, super Käler geometry and geometric quantization," Communications in Mathematical Physics, vol. 175, no. 3, pp. 521-563, 1996.

[17] Q. Gu and D. Han, "Super-wavelets and decomposable wavelet frames," The Journal of Fourier Analysis and Applications, vol. 11, no. 6, pp. 683-696, 2005.

[18] A. Khrennikov and A. Yu, "The Hilbert super space," Soviet Physics-Doklady, vol. 36, pp. 759-760, 1991.

[19] R. Oliver, "Super Hilbert spaces," Communications in Mathematical Physics, vol. 214, no. 2, pp. 449-467, 2000.

[20] L. Zang, W. Sun, and D. Chen, "Excess of a class of $g$-frames," Journal of Mathematical Analysis and Applications, vol. 352, no. 2, pp. 711-717, 2009.

[21] A. Abdollahi and E. Rahimi, "Generalized frames on super Hilbert spaces," Bulletin of the Malaysian Mathematical Sciences Society, vol. 35, no. 3, pp. 807-818, 2012.
[22] A. Najati, M. H. Faroughi, and A. Rahimi, " $G$-frames and stability of $g$-frames in Hilbert spaces," Methods of Functional Analysis and Topology, vol. 14, no. 3, pp. 271-286, 2008.

[23] W. Sun, "Stability of g-frames," Journal of Mathematical Analysis and Applications, vol. 326, no. 2, pp. 858-868, 2007.

[24] Y. J. Wang and Y. C. Zhu, "G-frames and g-frame sequences in Hilbert spaces," Acta Mathematica Sinica, vol. 25, no. 12, pp. 2093-2106, 2009. 


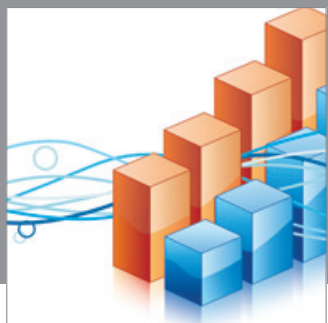

Advances in

Operations Research

mansans

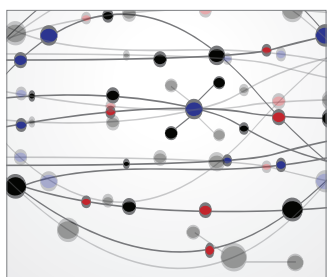

The Scientific World Journal
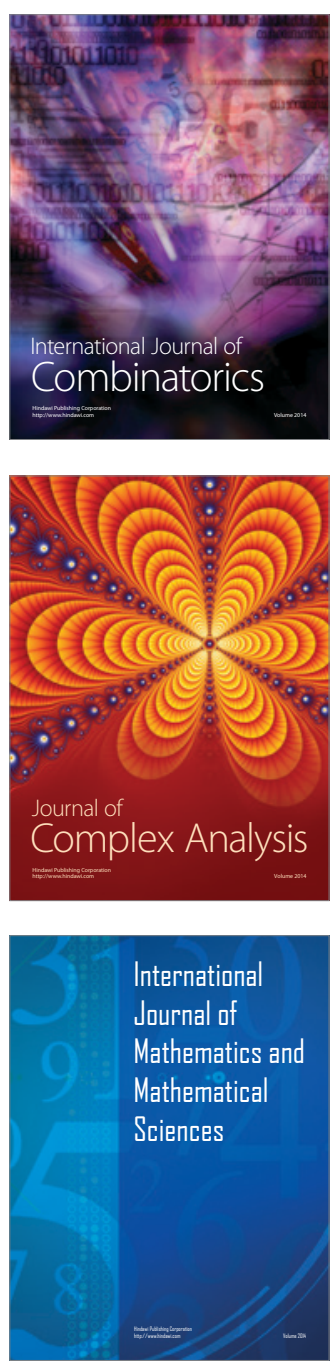
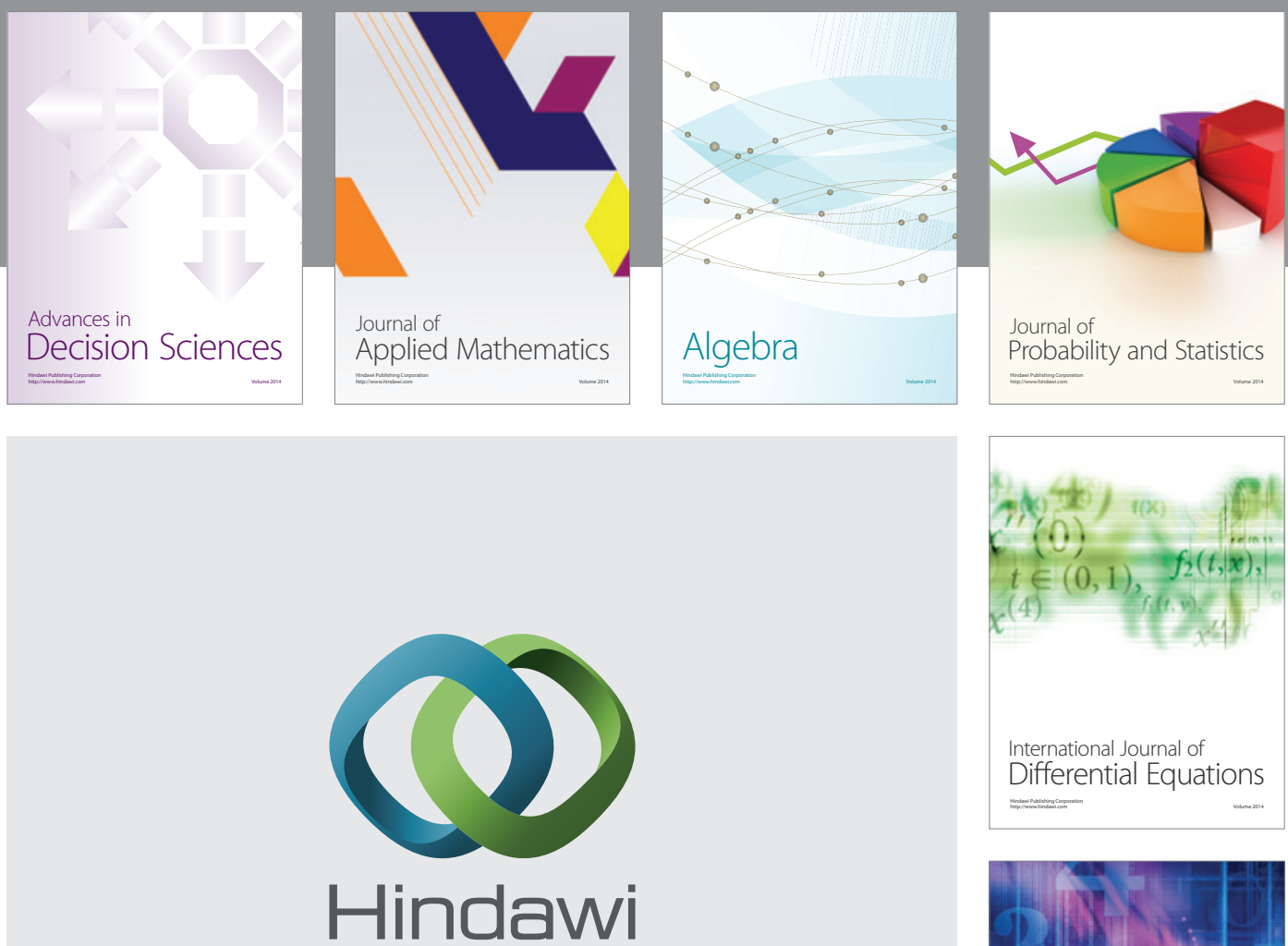

Submit your manuscripts at http://www.hindawi.com
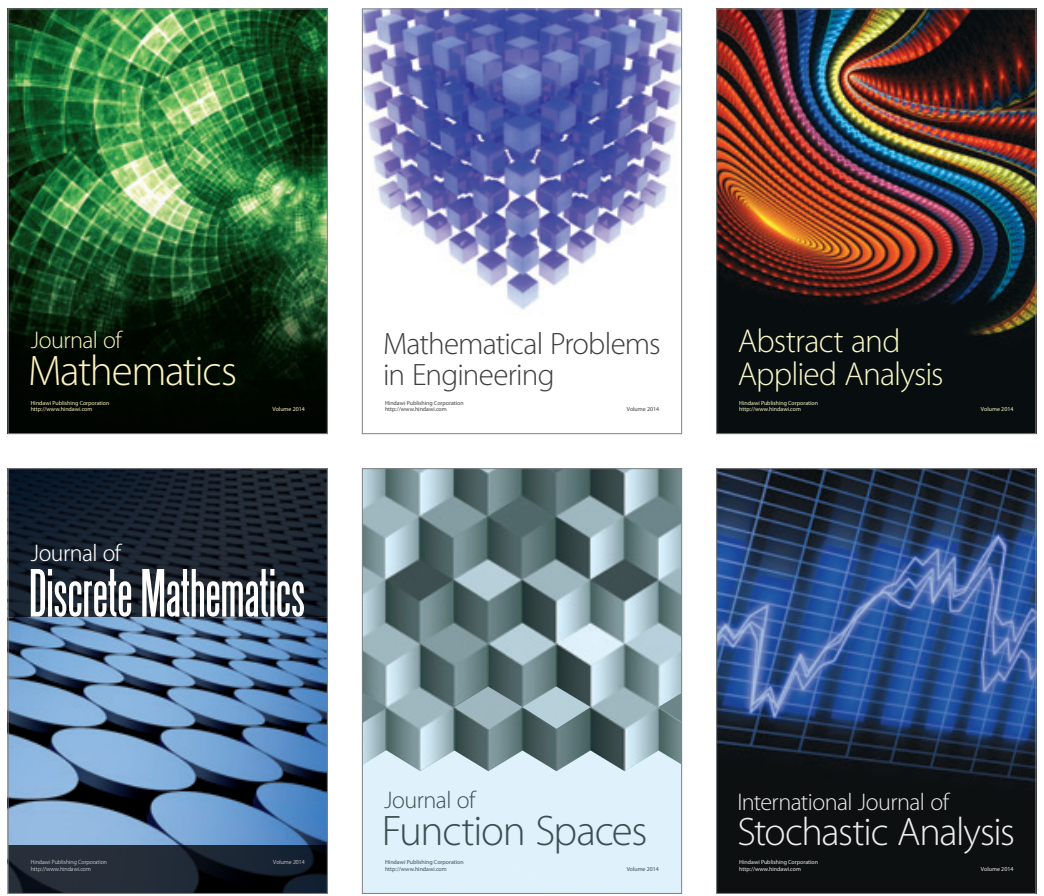

Journal of

Function Spaces

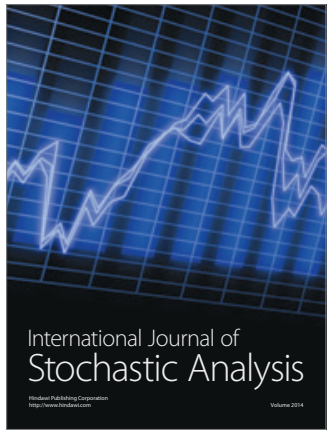

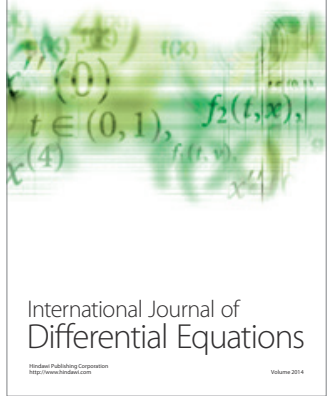
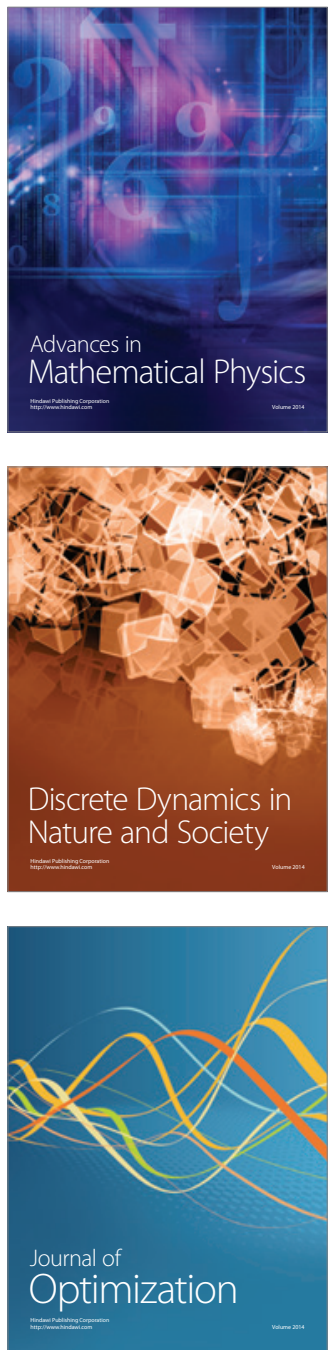